\title{
Right Hemispheric Predominance of Brain Infarcts in Atrial Fibrillation: A Lesion Mapping Analysis
}

\author{
Anna Altermatt, ${ }^{\mathrm{a}, \mathrm{b}, *}$ Tim Sinnecker, ${ }^{\mathrm{a}, \mathrm{c}, *}$ Stefanie Aeschbacher, ${ }^{\mathrm{d}, \mathrm{e}}$ Anne Springer, ${ }^{\mathrm{d}, \mathrm{e}}$ Michael Coslovsky, ${ }^{\mathrm{de}, \mathrm{f}}$ \\ Juerg Beer, ${ }^{\mathrm{g}}$ Giorgio Moschovitis, ${ }^{\mathrm{h}}$ Angelo Auricchio, ${ }^{\mathrm{i}}$ Urs Fischer, ${ }^{\mathrm{j}}$ Carole E. Aubert, ${ }^{\mathrm{k}, \mathrm{l}, \mathrm{m}, \mathrm{n}}$ \\ Michael Kühne, ${ }^{\mathrm{d}, \mathrm{e}}$ David Conen, ${ }^{\mathrm{o}}$ Stefan Osswald, ${ }^{\mathrm{d}, \mathrm{e}}$ Leo H. Bonati, ${ }^{\mathrm{c}}$ Jens Wuerfel, ${ }^{\mathrm{a}, \mathrm{b}, \mathrm{p}}$ for the Swiss-AF \\ Study Investigators \\ ${ }^{a}$ Medical Image Analysis Center (MIAC AG), Basel, Switzerland \\ ${ }^{b}$ Department of Biomedical Engineering, University of Basel, Basel, Switzerland \\ 'Department of Neurology, University Hospital Basel, Basel, Switzerland \\ ${ }^{\mathrm{d} C}$ Cardiology Division, Department of Medicine, University Hospital Basel, University of Basel, Basel, Switzerland \\ ${ }^{\text {e}}$ Cardiovascular Research Institute Basel, University Hospital Basel, University of Basel, Basel, Switzerland \\ ${ }^{f}$ Clinical Trial Unit, Department of Clinical Research, University Hospital Basel, University of Basel, Basel, Switzerland \\ ${ }^{9}$ Department of Medicine, Baden Cantonal Hospital, Baden, Switzerland \\ ${ }^{h}$ Division of Cardiology, Department of Medicine, Ente Ospedaliero Cantonale (EOC), Regional Hospital of Lugano, Lugano, Switzerland \\ 'Division of Cardiology, Fondazione Cardiocentro Ticino, Lugano, Switzerland \\ 'Department of Neurology, Inselspital, University Hospital of Bern, University of Bern, Bern, Switzerland \\ kDepartment of General Internal Medicine, Inselspital, University Hospital of Bern, Bern, Switzerland \\ 'Institute of Primary Health Care (BIHAM), University of Bern, Bern, Switzerland \\ ${ }^{m}$ Center for Clinical Management Research, Veterans Affairs Ann Arbor Healthcare System, Ann Arbor, MI, USA \\ nInstitute for Healthcare Policy and Innovation, University of Michigan, Ann Arbor, MI, USA \\ ${ }^{\circ}$ Population Health Research Institute, McMaster University, Hamilton, ON, Canada \\ ${ }^{p}$ NeuroCure Research Center, Charité University Medicine Berlin, Berlin, Germany \\ *These authors contributed equally to the manuscript as first author.
}

\section{Dear Sir:}

Atrial fibrillation (AF) increases the risk of an ischemic stroke three- to five-fold, ${ }^{1}$ and cardioembolic strokes are associated with worse outcomes than strokes of other origin. ${ }^{2}$ Covert brain infarcts occur in more than a third of patients with $\mathrm{AF}$ and have been associated with cognitive impairment. ${ }^{3}$ Regional distribution of brain infarcts may explain why most patients with $A F$ and brain infarcts on neuroimaging have no clinical history of stroke or transient ischemic attack (TIA). Previous studies on a possible side-predominance of brain infarction in patients with AF have yielded conflicting evidence and were mostly done in patients with clinically manifest stroke. ${ }^{4}$ In the present study, we systematically analyzed the anatomical distribution and localization of covert and overt brain infarcts according to different vascular territories in a large cohort study of patients with established AF.
This is a cross-sectional analysis of the Swiss-AF cohort, a prospective, multicenter cohort study of unselected patients with AF across 14 centers in Switzerland. ${ }^{5}$ In total, the study has enrolled 2,415 participants with AF. At baseline visits, brain magnetic resonance imaging (MRI) was acquired in all patients (without contraindications for MRI) on either a 1.5 or a 3.0 Tesla scanner, depending on the participating site. The most frequent reason for missing MRI was the presence of an implanted cardiac device $(n=461)$. The 1,716 patients with eligible baseline brain MRI data (mean age $73 \pm 8$ years, $27 \%$ women, $20 \%$ with history of stroke or TIA) were included in the present study (Supplementary Table 1). Detailed methodological descriptions are provided in the Supplementary methods. ${ }^{3,5-11}$ Briefly, we manually segmented brain infarcts on MRI. Lesions were classified into large non-cortical or cortical infarcts (LNC$\mathrm{Cls}$ ) and small non-cortical infarcts (SNCls). ${ }^{7}$ LNCCls were considered infarcts of potentially embolic or atherothrombotic ori- 

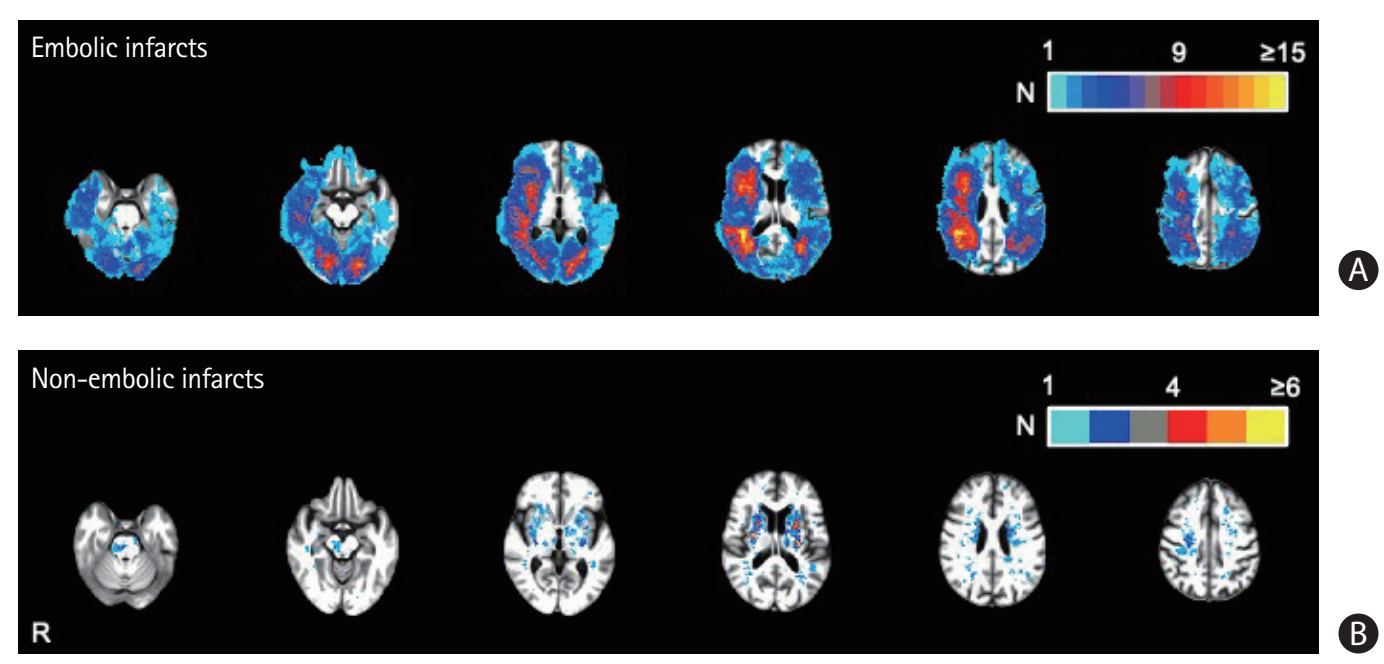

Figure 1. The distribution of infarcts. Figure depicting the voxel-based sum of all large cortical and non-cortical infarcts and small non-cortical infarcts overlaid on an age-specific standard brain template. The color scale indicates the number of patients with an infarct in a given voxel.

gin and included large non-cortical infarcts with a diameter of $>20 \mathrm{~mm}$, and cortical infarcts of any size. SNCls were considered infarcts of potentially microangiopathic origin and defined as lesions with a diameter of $\leq 20 \mathrm{~mm}$ not involving the cortex. Lesion masks were co-registered separately to an age-specific brain template and overlaid to compute a voxel-based probability map.

We observed LNCCls in 386 (22\%) patients. Visually, in the voxel-based probability map, a clear right-hemispheric preponderance was observed (Figure 1A). Results of the statistical comparison of lesion counts, average lesion volumes, and percentages affected per vascular territory are reported in Supplementary Table 2. LNCCls were most often located in the territory of the middle cerebral artery (MCA). While, in the MCA territory, LNCCI counts were evenly distributed between the hemispheres (Wilcoxon matched-pair rank test; $P=0.796$ ), the average volume of infarcts was significantly larger in the right compared to the left hemisphere (left [total]: $512.4 \mathrm{~mL}$ vs. right [total]: 1,240.1 mL; Wilcoxon matched-pair rank test; $P=0.002$ ). Similarly, the percentage of the MCA territory affected by LNC$\mathrm{Cls}$ (calculated by the proportion of lesional voxels per vascular territory per patient) was significantly larger in the right than in the left hemisphere (Wilcoxon matched-pair rank test; $P=0.008$ ). No side-preponderance of $\mathrm{LNCCls}$ was detected for the vascular territories of the anterior and posterior cerebral arteries. No side-difference was observed for SNCls (Figure 1B).

Our findings might indicate, that large emboli preferentially hit the right brain hemisphere. We hypothesize that both vascular anatomy (i.e., the right artery originating more proximally from the heart) and the physical properties of cardiac emboli (i.e. their size) contribute to the right-sided preponderance for embolic cerebral infarcts. The right common carotid artery originates more proximally from the heart and with less angulation from the aortic arch, which in turn increases the likelihood of these large emboli to enter the right carotid artery. This may especially be the case for larger cardiac emboli which are dragged to the outer curvature of the aorta. This hypothesis is supported by a study using anatomically accurate models of the human aorta and their branch arteries. ${ }^{12}$

As no information on competing stroke etiologies was collected in SWISS-AF, we cannot know how many LNCCls might have been of potential atheroembolic origin, which represents a major limitation of our study. However, it is unlikely that the observed asymmetry in LNCCls is explained by atherosclerosis of the aortic arch or supra-aortic arteries.

A better understanding of the localization in relation to the size of brain infarcts might be helpful in clinical routine. Focal symptoms and signs originating from right hemispheric lesions may easily be missed by patients and physicians. The proportion of patients with a history of clinically manifest stroke or TIA was consistent with previous population-based studies in $\mathrm{AF}^{1}{ }^{1}$ but $37,1 \%$ of patients had an ischemic infarct on MRI ( $\mathrm{SNCl}$ or $\mathrm{LNCCl}$ combined). We have previously shown that covert brain infarcts have a significant impact on cognitive decline in $\mathrm{AF}_{1}^{3}$ and are associated with an increased risk of future strokes. ${ }^{13}$ Whether routine brain MRI screening in patients with AF improves risk stratification remains to be elucidated by future prospective studies.

\section{Supplementary materials}

Supplementary materials related to this article can be found 
online at https://doi.org/10.5853/jos.2021.03531.

\section{References}

1. Wolf PA, Abbott RD, Kannel WB. Atrial fibrillation as an independent risk factor for stroke: the Framingham Study. Stroke 1991;22:983-988.

2. Kamel H, Healey JS. Cardioembolic stroke. Circ Res 2017;120: 514-526.

3. Conen D, Rodondi N, Müller A, Beer JH, Ammann P, Moschovitis $G$, et al. Relationships of overt and silent brain lesions with cognitive function in patients with atrial fibrillation. $J$ Am Coll Cardiol 2019;73:989-999.

4. Kim HJ, Song JM, Kwon SU, Kim BJ, Kang DH, Song JK, et al. Right-left propensity and lesion patterns between cardiogenic and aortogenic cerebral embolisms. Stroke 2011;42:23232325.

5. Conen D, Rodondi N, Mueller A, Beer J, Auricchio A, Ammann $P$, et al. Design of the Swiss Atrial Fibrillation Cohort Study (Swiss-AF): structural brain damage and cognitive decline among patients with atrial fibrillation. Swiss Med Wkly 2017; 147:w14467.

6. Kappos L, Radue EW, O'Connor P, Polman C, Hohlfeld R, Calabresi $\mathrm{P}_{\text {, et al. }}$ A placebo-controlled trial of oral fingolimod in relapsing multiple sclerosis. N Engl J Med 2010;362:387-401.

7. Wardlaw JM, Smith EE, Biessels GJ, Cordonnier C, Fazekas F, Frayne $R$, et al. Neuroimaging standards for research into small vessel disease and its contribution to ageing and neurodegeneration. Lancet Neurol 2013;12:822-838.

8. Battaglini M, Jenkinson M, De Stefano N. Evaluating and reducing the impact of white matter lesions on brain volume measurements. Hum Brain Mapp 2012;33:2062-2071.

9. Jenkinson M, Smith S. A global optimisation method for robust affine registration of brain images. Med Image Anal 2001;5: 143-156.

10. Andersson JLR, Jenkinson M, Smith S. Non-Linear Registration Aka Spatial Normalisation. FMRIB Technial Report TR07JA2. Oxford, UK: FMRIB Center, 2007. https://www.fmrib. ox.ac.uk/datasets/techrep/tr07ja2/tr07ja2.pdf: Assessed January 11, 2022.

11. Schirmer MD, Giese AK, Fotiadis $P$, Etherton MR, Cloonan L, Viswanathan $A$, et al. Spatial signature of white matter hyperintensities in stroke patients. Front Neurol 2019;10:208.

12. Carr IA, Nemoto N, Schwartz RS, Shadden SC. Size-dependent predilections of cardiogenic embolic transport. Am J Physiol Heart Circ Physiol 2013;305:H732-H739.

13. Gupta A, Giambrone AE, Gialdini G, Finn C, Delgado D, Gutierrez $J$, et al. Silent brain infarction and risk of future stroke: a systematic review and meta-analysis. Stroke 2016;47:719725.

Correspondence: Leo $\mathrm{H}$. Bonati

Department of Neurology, University Hospital Basel, CH-4031 Basel, Switzerland Tel: +41-61-265-2525

Fax: +41-61-265-2020

E-mail: leo.bonati@usb.ch

https://orcid.org/0000-0003-1163-8133

Received: October 8, 2021

Revised: January 7, 2022

Accepted: January 11, 2022

Anna Altermatt is employee of the Medical Image Analysis Center Basel, Switzerland; Tim Sinnecker is employee of the Medical Image Analysis Center Basel, Switzerland; Stefanie Aeschbacher: none; Anne Springer: none; Michael Coslovsky: none; Juerg Beer reports grants from the Swiss National Foundation of Science, The Swiss Heart Foundation, grants from Bayer, lecture fees from Sanofi Aventis and Amgen, to the institution outside the submitted work; Giorgio Moschovitis reports consultant fees for participating to advisory boards from Novartis, Astra Zeneca, Bayer, and Böhringer Ingelheim, outside of the current work; Angelo Auricchio is a consultant to Boston Scientific, Backbeat, Biosense Webster, Cairdac, Corvia, Microport CRM, EPD-Philips, Radcliffe Publisher. He received speaker fees from Boston Scientific, Medtronic, and Microport. He participates in clinical trials sponsored by Boston Scientific, Medtronic, EPD-Philips. He has intellectual properties with Boston Scientific, Biosense Webster, and Microport CRM; Urs Fischer received research grants for the Swiss National Science Foundation, Swiss Heart Foundation, Medtronic; Consultant for Stryker, Medtronic and CSL Behring; Carole E. Aubert was supported by an Early Postdoc.Mobility grant from the Swiss National Science Foundation (grant P2LAP3_184042); Michael Kühne reports personal fees from Bayer, personal fees from Böhringer Ingelheim, personal fees from Pfizer BMS, personal fees from Daiichi Sankyo, personal fees from Medtronic, personal fees from Biotronik, personal fees from Boston Scientific, personal fees from Johnson\&Johnson, personal fees from Roche, grants from Bayer, grants from Pfizer, grants from Boston Scientific, grants from BMS, grants from Biotronik; David Conen speaker fees from BMS/ Pfizer, and consultancy fees from Roche Diagnostics, Switzerland, both outside of the current work; Stefan Osswald: none; Leo H. Bonati received grants from the Swiss National Science Foundation, the Swiss Heart Foundation, and the University of Basel. He received consultancy and advisory board fees from Bayer and Bristol-Myers Squibb; and Jens Wuerfel is employee of the Medical Image Analysis Center (MIAC AG) Basel, Switzerland. He is or was supported by grants of the EU (Horizon2020), the Swiss National Science Foundation, the Boehringer Ingelheim Foundation, the Novartis Foundation, the German Federal Ministries of Education and Research (BMBF) as well as Economic Affairs and Energy (BMWI). He has served on advisory boards of Actelion, Apellis, Bayer, Biogen, Cellgene, Genzyme-Sanofi, Idorsia, InmuneBio, Novartis, and Roche.

The Swiss-AF study is supported by grants of the Swiss National Science Foundation (Grant numbers 33CS30_148474, 33CS30_177520, 32473B_176178, 32003B_197524), the Swiss Heart Foundation, the Foundation for Cardiovascular Research Basel, the Foundation for gastroenterological, general clinical and imaging research and analysis, Basel, the Medical Image Analysis Center (MIAC AG) Basel, and the University of Basel.

\section{Swiss-AF investigators}

University Hospital Basel and Basel University: Stefanie Aeschbacher, Katalin Bhend, Steffen Blum, Leo Bonati, David Conen, Ceylan Eken, Urs Fischer, Corinne Girroy, Elisa Hennings, Elena Herber, Vasco Iten, Philipp Krisai, Michael Kühne, Maurin Lampart, Mirko Lischer, Nina Mäder, Christine Meyer-Zürn, Pascal Meyre, Andreas U. Monsch, Luke Mosher, Christian Müller, Stefan Osswald, Rebecca E. Paladini, Anne Springer, Christian Sticherling, Thomas Szucs, Gian Völlmin; Principal Investigator: Stefan Osswald; Local principal investigator: Michael Kühne. University Hospital Bern: Faculty (Drahomir Aujesky, Juerg Fuhrer, Laurent Roten, Simon Jung, Heinrich Mattle); Research fellows (Seraina Netzer, Luise Adam, 
Carole Elodie Aubert, Martin Feller, Axel Loewe, Elisavet Moutzouri, Claudio Schneider); Study nurses (Tanja Flückiger, Cindy Groen, Lukas Ehrsam, Sven Hellrigl, Alexandra Nuoffer, Damiana Rakovic, Nathalie Schwab, Rylana Wenger, Tu Hanh Zarrabi Saffari); Local principal investigator: Nicolas Rodondi, Tobias Reichlin.

Stadtspital Triemli Zurich: Christopher Beynon, Roger Dillier, Michèle Deubelbeiss, Franz Eberli, Christine Franzini, Isabel Juchli, Claudia Liedtke, Samira Murugiah, Jacqueline Nadler, Thayze Obst, Jasmin Roth, Fiona Schlomowitsch, Xiaoye Schneider, Katrin Studerus, Noreen Tynan, Dominik Weishaupt; Local principal investigator: Andreas Müller.

Kantonspital Baden: Simone Fontana, Corinne Friedli, Silke Kuest, Karin Scheuch, Denise Hischier, Nicole Bonetti, Alexandra Grau, Jonas Villinger, Eva Laube, Philipp Baumgartner, Mark Filipovic, Marcel Frick, Giulia Montrasio, Stefanie Leuenberger, Franziska Rutz; Local principal investigator: Jürg-Hans Beer.

Cardiocentro Lugano: Angelo Auricchio, Adriana Anesini, Cristina Camporini, Giulio Conte, Maria Luce Caputo, Francois Regoli; Local principal investigator: Tiziano Moccetti.

Kantonsspital St. Gallen: Roman Brenner, David Altmann, Michaela Gemperle; Local principal investigator: Peter Ammann.

Hôpital Cantonal Fribourg: Mathieu Firmann, Sandrine Foucras, Martine Rime; Local principal investigator: Daniel Hayoz.

Luzerner Kantonsspital: Benjamin Berte, Virgina Justi, Frauke Kellner-Weldon, Brigitta Mehmann, Sonja Meier, Myriam Roth, Andrea Ruckli-Kaeppeli, lan Russi,
Kai Schmidt, Mabelle Young, Melanie Zbinden; Local principal investigator: Richard Kobza.

Ente Ospedaliero Cantonale Lugano: Elia Rigamonti, Carlo Cereda, Alessandro Cianfoni, Maria Luisa De Perna, Jane Frangi-Kultalahti, Patrizia Assunta Mayer Melchiorre, Anica Pin,Tatiana Terrot, Luisa Vicari; Local principal investigator: Giorgio Moschovitis.

University Hospital Geneva: Georg Ehret, Hervé Gallet, Elise Guillermet, Francois Lazeyras, Karl-Olof Lovblad, Patrick Perret, Philippe Tavel, Cheryl Teres; Local principal investigator: Dipen Shah,

University Hospital Lausanne: Nathalie Lauriers, Marie Méan, Sandrine Salzmann, Jürg Schläpfer; Local principal investigator: Alessandra Pia Porretta.

Bürgerspital Solothurn: Andrea Grêt, Jan Novak, Sandra Vitelli; Local principal investigator: Frank-Peter Stephan.

Ente Ospedaliero Cantonale Bellinzona: Jane Frangi-Kultalahti, Augusto Gallino, Luisa Vicari; Local principal investigator: Marcello Di Valentino.

University of Zurich/University Hospital Zurich: Helena Aebersold, Fabienne Foster, Matthias Schwenkglenks.

Medical Image Analysis Center AG Basel: Jens Würfel (Head), Anna Altermatt, Michael Amann, Marco Düring, Petra Huber, Esther Ruberte, Tim Sinnecker, Vanessa Zuber.

Clinical Trial Unit Basel: Michael Coslovsky (Head), Pascal Benkert, Gilles Dutilh, Milica Markovic, Pia Neuschwander, Patrick Simon.

Schiller AG Baar: Ramun Schmid. 


\section{Supplementary methods}

\section{Study population}

The Swiss Atrial Fibrillation (Swiss-AF) study is a prospective cohort study which has enrolled 2,415 participants with established atrial fibrillation (AF) in 14 centers in Switzerland. The detailed study design and first results have been reported previously. 3.5 Included patients were $\geq 65$ years old and had either paroxysmal AF defined as: self-terminating AF lasting $<7$ days that does not require cardioversion and that was documented at least twice within the last 60 months; persistent AF defined as AF sustained $\geq 7$ days and/or re-quiring cardioversion, documented within the last 60 months; or permanent AF. Additionally, a smaller group of patients between 45 and 65 years of age was included to assess the effects of AF on actively employed individuals. Patients with a secondary form of AF or unable to provide informed consent were excluded.

\section{Standard protocol approvals, registrations, and patient consents}

The study protocol was approved by the local ethics committees (Ethikkommission Nordwest- und Zentralschweiz), and written informed consent was obtained from all participants (Trial Registration: ClinicalTrials.gov Identifier, NCT02105844).

\section{Image acquisition}

Baseline brain magnetic resonance imaging (MRI) was acquired on either a 1.5 or a 3.0 Tesla scanner, depending on the participating site. The standardized protocol has been described in detail previously. ${ }^{3,5}$ Of relevance for the present study, 3D T1-weighted (T1w) magnetization prepared rapid gradient echo (MPRAGE; spatial resolution $1.0 \times 1.0 \times 1.0 \mathrm{~mm}^{3}$ ), and $2 \mathrm{D}$ axial T2-weighted fluid attenuated inversion recovery (FLAIR; spatial resolution $1.0 \times 1.0 \times 3.0 \mathrm{~mm}^{3}$ ) sequences. Out of the $2,415 \mathrm{pa}-$ tients enrolled in Swiss-AF, complete baseline MRI was available in 1,748 patients. The most frequent reason for missing MRI was the presence of an implanted cardiac device $(n=461$, $69 \%)$.

\section{Lesion segmentation}

Lesion segmentation was performed on FLAIR images by professional medical image analysts at the Medical Image Analysis Center (MIAC AG, Basel, Switzerland), blinded to clinical and other information, according to in-house standard operating procedures approved for international clinical phase III trials. ${ }^{6}$ Board certified neuroradiologists confirmed all ratings. Lesions were classified into large non-cortical or cortical infarcts (LNC$\mathrm{Cls}$ ) and small non-cortical infarcts (SNCls). LNCCls were con- sidered infarcts of potentially embolic origin and included (1) large non-cortical infarcts with a diameter of $>20 \mathrm{~mm}$, and (2) cortical infarcts defined as hyperintense lesions on FLAIR involving the cortex irrespective of their size. SNCls were defined as hyperintense lesions on FLAIR $\leq 20 \mathrm{~mm}$ in diameter assessed on axial sections and not involving the cortex. ${ }^{7}$

\section{Image registration}

To prepare images for image co-registration, the patients' brain data were first extracted from the T1w images using FreeSurfer v6.0 (binarization of 'aseg.auto' output from 'recon-all' pipeline; https://surfer.nmr.mgh.harvard.edu/fswiki). Resulting brain masks were dilated slice-by-slice by two voxels since less restrictive brain extraction yielded better results in the later registration. Secondly, corresponding T2w FLAIR white matter hyperintense lesions and LNCCls were filled on T1w images to minimize their effect on image registration. This was done using the lesion filling algorithm encoded in $\mathrm{FSL}^{5}$ and masking of the lesion portions in the grey matter.

For image registration (FSL v5.0, FMRIB's Software Library; https://fsl.fmrib.ox.ac.uk/fsl/fslwiki), FLAIR images were first linearly registered to T1w images using FSL (FMRIB's linear registration tool, FLIRT; using defaults settings, 6 degrees of freedom). ${ }^{8,9}$ The transformation parameters resulting from this image registration were then applied to the $\mathrm{SNCl}$ and $\mathrm{LNCCl}$ lesion masks (FLAIR image contrast) using a nearest-neighbor interpolation. At this step, the skull was removed from the FLAIR-to-T1w images using the previously prepared brain masks. Then, FLAIR-to-T1w images were non-linearly registered to an age-specific standard brain template from the BRAINS image bank (https://www.brainsimagebank.ac.uk/; for brains early to mid-seventies; spatial resolution $1.0 \times 1.3 \times 1.0$ $\mathrm{mm}^{3}$ ) using FSL (FMRIB's non-linear registration tool, FNIRT; settings as specified in the provided configuration file, taking as reference the age-specific brain template). ${ }^{10}$ The resulting transformation parameters were then applied to the transformed FLAIR-to-T1w lesion masks again using nearest-neighbor interpolation. The quality assessment of the registration was done by trained and experienced raters. If images showed strong distortions in the registered images, patients were excluded from subsequent analyses. At image registration, 32 patients were excluded, leaving 1,716 patients (98\%) for the present analyses.

\section{Spatial lesion distribution and mapping onto vascular territories}

The co-registered $\mathrm{LNCCl}$ and SNCl lesion masks were overlaid on the age-specific brain template to generate voxel-based le- 
sion probability maps. Lesion masks were then projected onto the co-registered MNI Vascular Territories Atlas. ${ }^{11}$ Number and volume of lesions within anterior cerebral artery (ACA), middle cerebral artery (MCA), and posterior cerebral artery (PCA) territories, were computed. Moreover, the percentages of vascular territories affected by LNCCls or SNCls (i.e., percentage overlay) were calculated.

\section{Statistical analyses}

Infarct counts, volumes, and percentages were reported. We tested if these measures differed within vascular territories of left and right brain hemispheres. The Wilcoxon matched-pair rank test was used to compare median differences of infarct counts, volumes, and percentages within ACA, MCA, and PCA. Only patients having an infarct in this specific vascular territory-either in the left or the right hemisphere-were considered. No correction of $P$-values for multiple testing was performed due to the exploratory nature of the analysis. All statistical analyses were done in $\mathrm{R}$ version 3.6.3. (https://www.r-project. org/).

\section{Data availability statement}

The patient informed consent forms, as approved by the responsible ethics committee, do not allow the data to be made publicly available. The participants signed a consent form, which states that their data, containing personal and medical information, are exclusively available for research institutions in an anonymized form. Researchers interested in obtaining the data for research purposes can contact the Swiss-AF scientific lead. Contact information is provided on the Swiss-AF website (http://www.swissaf.ch/contact.htm). Authorization of the responsible ethics committee is mandatory before the requested data can be transferred to external research institutions. 
Supplementary Table 1. Baseline characteristics of patients

\begin{tabular}{lc}
\hline Variable & Value \\
\hline Age (yr) & $72.5 \pm 8.4$ \\
Female sex & $471(27.4)$ \\
Comorbidities & $1,186(69.1)$ \\
History of hypertension & $267(15.6)$ \\
History of diabetes & $228(13.3)$ \\
History of stroke & $343(20.0)$ \\
History of stroke/TIA & $455(26.5)$ \\
History of CAD & $122(7.1)$ \\
History of PVD & $234(13.6)$ \\
History of sleep apnea & \\
Oral anticoagulation & $922(53.7)$ \\
NOAC & $625(36.4)$ \\
VKA & \\
\hline
\end{tabular}

Values are presented as mean \pm standard deviation or number $(\%)$.

$T I A$, transient ischemic attack; CAD, coronary artery disease; PVD, peripheral vascular disease; NOAC, non-vitamin K oral anticoagulant; VKA, vitamin K antagonist.

Supplementary Table 2. Infarcts per vascular territory in all patients

\begin{tabular}{|c|c|c|c|c|c|c|c|c|c|c|c|c|c|}
\hline \multirow{2}{*}{ LNCCI } & \multicolumn{2}{|c|}{ Total count } & \multicolumn{3}{|c|}{ Count } & \multicolumn{2}{|c|}{ Total volume (mL) } & \multicolumn{3}{|c|}{ Infarct volume $(\mathrm{mL})$} & \multicolumn{3}{|c|}{ Percentage } \\
\hline & Left & Right & Left & Right & $P$ & Left & Right & Left & Right & $P$ & Left & Right & $P$ \\
\hline ACA & 229 & 248 & $1(0-11)$ & $1(0-16)$ & 0.999 & 138.5 & 91.1 & $0(0-17.5)$ & $0(0-3.8)$ & 0.279 & $0.0(0-11.8)$ & $0.0(0-6.3)$ & 0.367 \\
\hline MCA & 449 & 438 & $1(0-23)$ & $1(0-23)$ & 0.1 & 4 & 1 & 0 & 0.2 & 0.002 & 3.3) & 27.1) & 0.008 \\
\hline PCA & 383 & 426 & $1(0-30)$ & $1(0-34)$ & 0.996 & 207.5 & 208.8 & $0(0-4.1)$ & $0(0-10.8)$ & 0.872 & $0.0(0-26.0)$ & $0.0(0-24.7)$ & 0.834 \\
\hline
\end{tabular}

Values are presented as median (interquartile range). Number and volume of infarcts within left or right ACA, MCA, and PCA are shown. The variable 'volume' corresponds to the total infarct volume per vascular territory in all patients. The variable 'infarct volume' refers to the average infarct volume within a vascular territory (if there are multiple infarcts per patient and territory). Moreover, the percentages of vascular territories affected by the infarcts is reported (corresponds to the proportion of lesional voxels per vascular territory per patient). The Wilcoxon matched-pair rank test was used to compare median differences of infarct counts, volumes, and percentages between hemispheres. Only patients having an infarct in this specific vascular territory-either in the left or the right hemisphere-were considered.

LNCCI, large non-cortical or cortical infarct; ACA, anterior cerebral artery; MCA, middle cerebral artery; PCA, posterior cerebral artery. 